\title{
A missense variant impairing TRMT1 function in tRNA modification is linked to intellectual disability
}

Kejia Zhang ${ }^{1}$, Jenna M Lentini ${ }^{1}$, Christopher T Prevost ${ }^{1}$, Mais O Hashem ${ }^{2,3}$, Fowzan S Alkuraya ${ }^{2,3}$, and Dragony $\mathrm{Fu}^{1 *}$

${ }^{1}$, Department of Biology, Center for RNA Biology, University of Rochester, Rochester, NY 14627

${ }^{2}$, Department of Genetics, King Faisal Specialist Hospital and Research Center, Riyadh, Saudi Arabia

${ }^{3}$, Department of Anatomy and Cell Biology, College of Medicine, Alfaisal University, Riyadh, Saudi Arabia

* corresponding author: Dragony Fu, dragonyfu@rochester.edu

ORCID: https://orcid.org/0000-0002-8725-8658

Keywords: tRNA modification, TRMT1, dimethylguanosine, intellectual disability 


\begin{abstract}
The human TRMT1 gene encodes a tRNA methyltransferase enzyme responsible for the formation of the dimethylguanosine $(\mathrm{m} 2,2 \mathrm{G})$ modification in cytoplasmic and mitochondrial tRNAs. Frameshift mutations in the TRMT1 gene have been shown to cause autosomal-recessive intellectual disability (ID) in the human population but additional TRMT1 variants remain to be characterized. Moreover, the impact of ID-associated TRMT1 mutations on m2,2G levels in IDaffected patients is unknown. Here, we describe a homozygous missense variant in TRMT1 in a patient displaying developmental delay, ID, and epilepsy. The missense variant changes a conserved arginine residue to a cysteine (R323C) within the methyltransferase domain of TRMT1 and is expected to perturb protein folding. Patient cells expressing the TRMT1-R323C variant exhibit a severe deficiency in $\mathrm{m} 2,2 \mathrm{G}$ modifications within tRNAs, indicating that the mutation causes loss-of-function. Notably, the TRMT1 R323C mutant retains the ability to bind tRNA but is unable to rescue $\mathrm{m} 2,2 \mathrm{G}$ formation in TRMT1-deficient human cells. Our results identify a pathogenic point mutation in TRMT1 that severely perturbs tRNA modification activity, and provide the first demonstration that $\mathrm{m} 2,2 \mathrm{G}$ modifications are disrupted in patients with TRMT1associated ID disorders.
\end{abstract}




\section{Introduction}

The post-transcriptional modification of tRNA has emerged as a critical modulator of biological processes ranging from gene expression to development (Frye et al. 2018; Ranjan and Leidel 2019). There are over 100 types of tRNA modifications that range from simple methylation to complex modifications involving multiple chemical groups (El Yacoubi et al. 2012; Ontiveros et al. 2019). Notably, defects in tRNA modification have emerged as the cause of diverse neurological and neurodevelopmental disorders, thereby highlighting the critical role of tRNA modification in human health and physiology (Angelova et al. 2018; Ramos and Fu 2018). In particular, the brain appears to be exquisitely sensitive to any perturbation in translation efficiency and fidelity brought about by defects in tRNA modifications, as evidenced from the numerous cognitive disorders linked to tRNA modification (Abbasi-Moheb et al. 2012; Alazami et al. 2013; Blanco et al. 2014; de Brouwer et al. 2018; El-Hattab et al. 2016; Khan et al. 2012; Komara et al. 2015; Martinez et al. 2012; Monies et al. 2019; Ramos et al. 2019; Shaheen et al. 2015; Shaheen et al. 2016a; Shaheen et al. 2016b; Shaheen et al. 2019a; Shaheen et al. 2019b).

The methylation of the nucleotide base or sugar by $S$-adenosyl-methionine (SAM)dependent methyltransferases represents one of the most common post-transcriptional modifications in tRNA (Ayadi et al. 2019; Hori 2014). One of the very first tRNA methyltransferase enzymes to be discovered is the tRNA methyltransferase 1 (Trm1p) enzyme from yeast Saccharomyces cerevisiae (Hopper et al. 1982). S. cerevisiae Trm1p is imported into the nucleus and mitochondria, where it catalyzes the methylation of a specific guanosine residue at position 26 in numerous tRNAs to yield the N2,N2-dimethylguanosine (m2,2G) modification (Ellis et al. 1987, 1989; Ellis et al. 1986). While S. cerevisiae strains lacking Trm1 are viable, Trm1-null strains display temperature sensitivity and defects in tRNA stability that are further exacerbated by combined deletion of either the Trm4p tRNA methyltransferase or the La RNA binding protein (Copela et al. 2006; Dewe et al. 2012). Furthermore, studies in Schizosaccahromyces pombe have identified particular tRNAs that are dependent upon Trm1p and/or La for proper folding, 
aminoacylation and accumulation (Vakiloroayaei et al. 2017). These observations highlight a key role for Trm1p-catalyzed m2,2G modifications in the stabilization of tRNA structure and function.

Two human homologs of yeast Trm1p have been identified by sequence homology that are encoded by the TRMT1 and TRMT1L genes (Buckland et al. 1996; Liu and Straby 2000; Vauti et al. 2007). While the substrates of TRMT1L remain to be discovered, TRMT1 has been demonstrated to be responsible for the majority of $m 2,2 \mathrm{G}$ modifications in the tRNA of human cells (Dewe et al. 2017). Notably, exome sequencing studies have implicated frameshift mutations in TRMT1 as the cause for certain forms of autosomal-recessive intellectual disability (ID) disorders (Blaesius et al. 2018; Davarniya et al. 2015; Monies et al. 2017; Najmabadi et al. 2011). The ID-associated TRMT1 mutants have been shown to be defective in tRNA binding and enzymatic activity (Dewe et al. 2017). While frameshift mutations in TRMT1 have been identified in the human population, TRMT1 missense alleles that help elucidate the functional consequences of tRNA modification deficiency remain to be found. Moreover, the extent to which $\mathrm{m} 2,2 \mathrm{G}$ modifications are impacted in patient cells by the TRMT1 mutations is unknown.

Here, we describe a TRMT1 missense variant in an individual presenting with ID disorder and epilepsy. Most significantly, we find that lymphoblastoid cells prepared from the patient exhibit a severe deficit in $\mathrm{m} 2,2 \mathrm{G}$ modifications in tRNAs. Moreover, we find that the mutant TRMT1 protein retains the ability to bind tRNA but is unable to rescue formation of $m 2,2 G$ in TRMT1deficient cell lines. Our results uncover a single amino acid substitution in TRMT1 that severely impairs tRNA modification function and provide evidence that the neurodevelopmental and cognitive defects caused by TRMT1 mutations are linked to loss of m2,2G modification.

\section{Results}

\section{Identification of a novel TRMT1 mutation implicated in ID}

We have previously described a "genomics first" approach to patients with ID (Anazi et al. 2017). A high yield of this approach was noted where a likely causal variant was identified in the 
majority of the $>330$ patients in that cohort. One of the reported variants in this cohort is a novel missense variant in the TRMT1 gene (NM_001136035.3 (TRMT1):c.967C>T, p.Arg323Cys) (Figure 1A). Subsequent Sanger sequencing confirmed the homozygous nature of the mutation in the ID-affected index individual (Figure 1B, 13DG1615). This female patient is currently 8 years old with ID, epilepsy and strabismus (Figure 1C). She was conceived via artificial insemination and pregnancy was uneventful. She was delivered at term vaginally but meconium aspiration resulted in respiratory distress and a short admission to neonatal intensive care for 5 days. Her motor development was normal but cognitive development was slow. Her IQ was 73 at the age of 6.5 years (Vineland scale), and she is currently attending special schooling with poor performance. Her medical history is notable for epilepsy that is partially controlled with medications and hearing loss (profound on the right and limited to peripheral auditory involvement on the left). Physical examination revealed normal brain MRI, head size, height and weight. However, she displayed subtle dysmorphism in the form of strabismus, epicanthus and smooth philtrum. The variant NM_001136035.3 (TRMT1):c.967C>T, p.Arg323Cys is completely absent in $>4,000$ Saudi exomes and is present at a very low frequency in gnomAD (4 heterozygotes, MAF 0.0000159). Moreover, the variant has a consistently deleterious prediction by in silico tools DANN, LRT, MutationAssessor, MutationTaster, PROVEAN, FATHMM-MKL and SIFT. These results identify the first missense mutation in the TRMT1 gene that is linked to a neurodevelopmental disorder.

\section{The R323C mutation is predicted to alter TRMT1 folding}

The ID-associated variant in the TRMT1 gene mutates amino acid reside 323 in the SAMmethyltransferase domain of TRMT1 from a positively charged arginine to a non-polar cysteine residue (R323C) (Figure 2A). Based upon protein sequence alignment, the R323 residue of human TRMT1 is absolutely conserved in Trm1 homologs from the Archaea to mammals (Figure 2B). Using the crystal structure of Trm1 from the Archaean Pyrococcus furiosus (Ihsanawati et al. 
2008), we found that the homologous arginine residue is located within the methyltransferase domain near the putative tRNA binding pocket within a four-stranded beta sheet (Figure 2C). Interestingly, the R323 residue is buried into the interior of Archaeal Trm1 rather than on the surface, unlike most charged side chains which are on the surface of proteins.

To gain insight into the potential effects of the R323C mutation on human TRMT1, we generated a predicted tertiary structure of human TRMT1 using an in silico template-based algorithm (Kallberg et al. 2012). Based upon this hypothetical structure, TRMT1 is predicted to fold into two distinct domains coinciding with the SAM-methyltransferase domain and the Cterminal CCCH-type Zinc finger motif (Figure 2D, TRMT1 methyltransferase domain). Notably, modeling of the R323C mutation using the most favored rotamer conformation would predict a steric clash with a conserved tyrosine side chain present at position 321 (Figure 2E). Thus, the R323C mutation is likely to be deleterious by perturbing the core packing of the methyltransferase domain of TRMT1 that is responsible for SAM binding, tRNA interaction and catalysis.

\section{Human patient cells homozygous for the R323C mutation exhibit a severe deficit in m2,2G modification in tRNA}

To examine the molecular effects of the R323C mutation, we generated a lymphoblastoid cell line (LCL) from the affected human patient harboring the homozygous missense mutation in the TRMT1 gene (referred to as R323C-LCL). The R323C-LCL was compared to control lymphoblasts generated from ethnically matched, healthy, unrelated individuals (WT-LCLs). We directly measured and compared the levels of more than 20 different tRNA modifications in the patient R323-LCL versus WT-LCLs through quantitative mass spectrometry of modified ribonucleosides derived from cellular RNA (Cai et al. 2015; Dewe et al. 2017). Strikingly, the m2,2G modification exhibited a 32-fold decrease in the R323-LCL compared to WT-LCL (Figure 3A). No other modification displayed a significant change between the WT versus R323-LCLs. 
To validate the perturbation of $\mathrm{m} 2,2 \mathrm{G}$ modification in cellular tRNAs, we used a primer extension assay that detects RNA modification status at nucleotide resolution. In this assay, the presence of $m 2,2 \mathrm{G}$ leads to a block of reverse transcriptase (RT) at position 26 of tRNA while a decrease in $\mathrm{m} 2,2 \mathrm{G}$ allows for read-through and an extended product up to a subsequent RTblocking modification. We selected three nuclear-encoded tRNAs that we have previously shown to contain m2,2G (Dewe et al. 2017), along with mitochondrial tRNA-Ile-GAU, which is the only known mammalian mitochondrial-encoded tRNA to contain m2,2G (Clark et al. 2016; Dewe et al. 2017; Suzuki and Suzuki 2014). In the absence of RT, only background bands were detected in reactions containing the probe and total cellular RNA from the wildtype LCL (Figure 3B, lane 1 for all tRNAs, background bands denoted by *). Addition of RT led to the appearance of an extension product up to the $\mathrm{m} 2,2 \mathrm{G}$ modification at the expected position in both nuclear- and mitochondrialencoded tRNAs in both WT-LCLs (Figure 3B, lanes 2 and 3 for tRNA-Ala-AGC and Ile-UAU or lanes 2 and 4 for tRNA-Met-CAU and mito-tRNA-Ile-UAU). In contrast, the RT block at position 26 was absent in the nuclear- and mitochondrial-encoded tRNA of the R323C-LCLs (Figure 3B, lane 4 for tRNA-Ala-AGC and Ile-UAU or lane 3 for tRNA-Met-CAU and mito-tRNA-Ile-UAU). Loss of $\mathrm{m} 2,2 \mathrm{G}$ modification in the tRNAs allowed for readthrough and extension to the next RT-blocking modification. Thus, LCLs from the ID-affected patient with the TRMT1 R323C mutation exhibit a severe defect in $\mathrm{m} 2,2 \mathrm{G}$ formation in cellular tRNAs.

\section{TRMT1 R323C mutant retains the ability to bind RNA but is unable to rescue $\mathrm{m} 2,2 \mathrm{G}$ formation in TRMT1-deficient cells}

To elucidate the molecular defects associated with the TRMT1-R323C mutant, we investigated the interaction between TRMT1 and tRNAs. As previously shown, human TRMT1 displays a stable interaction with substrate tRNAs that are targets for $m 2,2 \mathrm{G}$ modification (Dewe et al. 2017). Using this system, we expressed a FLAG-tagged version of TRMT1 variants in 293T human embryonic cells followed by affinity purification and analysis of copurifying RNAs. The 
expressed proteins represent either: 1) wildtype TRMT1, 2) the R323C mutant, and 3) Y445fs, a previously described, ID-associated TRMT1 mutant that lacks RNA binding due to the truncation of the RNA recognition motif (Figure 4A). Immunoblotting confirmed the expression and purification of each TRMT1 variant on anti-FLAG resin (Fig. 4B). In the control purification from vector-transfected cells, we detected only background contaminating 5.8S and 5S rRNAs (Figure 4C, lane 5). In contrast, the purification of WT-TRMT1 resulted in the considerable enrichment of tRNAs along with rRNAs as we have previously shown (Figure 4C, lane 6) (Dewe et al. 2017). Interestingly, we found that similar levels of tRNA were enriched with either TRMT1-WT or TRMT1-R323C mutant (Figure 4C, compare lanes 6 and 7). As expected, the Y445fs mutant exhibited only background RNA signal indicative of defective tRNA binding (Figure 4C, lane 8). Thus, the TRMT1-R323C mutant differs from other ID-associated TRMT1 variants by retaining the ability to bind tRNA.

We next used a previously-described TRMT1-knock out (KO) cell line derived from 293T human cells to dissect the effects of the R323C mutation on TRMT1 function (Dewe et al. 2017). This human 293T cell line lacks TRMT1 expression resulting in the near complete loss of m2,2G modifications in tRNA and the absence of $\mathrm{m} 2,2 \mathrm{G}$ modifications in all tested tRNAs. Using transient transfection of mammalian constructs, we expressed either WT-TRMT1 or the R323 variant in the WT or TRMT1-KO 293T cell lines (Figure 5A). We then accessed for rescue of $\mathrm{m} 2,2 \mathrm{G}$ formation in tRNA-Ala-AGC using the primer extension assay described above. As expected, nontransfected or vector-transfected WT 293T cells exhibited an RT block at position 26 of tRNA-AlaAGC indicative of the $\mathrm{m} 2,2 \mathrm{G}$ modification (Figure $5 \mathrm{~B}$ and $\mathrm{C}$, lanes 1 and 2 ). No readthrough product was detected for either tRNA in WT 293T cells suggesting that nearly all endogenous tRNA-Ala-AGC is modified with $\mathrm{m} 2,2 \mathrm{G}$. Consistent with this observation, increased expression of TRMT1 in WT 239T cells had no noticeable effect on $\mathrm{m} 2,2 \mathrm{G}$ modification in tRNA-Ala-AGC (Figure 5B, lane 3). Intriguingly though, over-expression of the TRMT1-R323C mutant in WT 293T cells led to increased readthrough product indicative of decreased $m 2,2 \mathrm{G}$ modification (Figure 
$5 B$, lane 4, quantified in $5 C$ ). The increase in read-through product suggests that TRMT1-R323C could have a dominant-negative effect on $\mathrm{m} 2,2 \mathrm{G}$ modification when over-expressed in the presence of WT-TRMT1.

We next tested the TRMT1-R323C mutant for the ability to rescue $\mathrm{m} 2,2 \mathrm{G}$ formation in the TRMT1-KO cell line. As expected, the m2,2G modification was absent in tRNA-Ala-AGC isolated from the non-transfected or vector-transfected TRMT1-KO cell line leading to readthrough to the next RT block (Figure 5B, lanes 5 and 6, quantified in $5 \mathrm{C}$ ). Re-expression of TRMT1-WT in the TRMT1-KO cell line was able to restore m2,2G formation (Figure 5B, lane 7 ). Due to variable TRMT1 expression caused by incomplete transfection efficiency, the level of $\mathrm{m} 2,2 \mathrm{G}$ modification was increased but not completely rescued to the level of the WT cell line. Notably, the TRMT1R323 mutant displayed greatly reduced ability to reconstitute m2,2G formation in the TRMT1-KO cell line (Figure 5B, lane 8, quantified in 5C). These results indicate that even though TRMT1R323C can retain binding to tRNAs, it is compromised in its capacity to generate $\mathrm{m} 2,2 \mathrm{G}$ in cellular tRNA. Thus, the TRMT1-R323C alteration appears to be a loss-of-function mutation, consistent with the severe deficiency in $\mathrm{m} 2,2 \mathrm{G}$ modification in the tRNAs of the affected patient with the R323C mutation.

\section{Discussion}

Here, we characterize a missense mutation in TRMT1 that causes a cognitive disorder characterized by ID and epilepsy. We further show that cells isolated from the patient with the TRMT1 R323C mutation exhibit a severe deficiency in $\mathrm{m} 2,2 \mathrm{G}$ modification within their tRNAs. The TRMT1-R323C mutant retains the ability to bind tRNA but is unable to rescue formation of tRNA modification. These results identify the first missense mutation in the TRMT1 mutation that causes ID and provide the first demonstration that $m 2,2 \mathrm{G}$ levels are impacted in individuals with a pathogenic TRMT1 mutation. 
Previously characterized TRMT1-ID mutations result in translation frameshifting that lead to truncation of the carboxyl-terminal zinc finger motif. These mutants have been shown to be defective in RNA binding and reconstitution of methyltransferase activity in vivo (Dewe et al. 2017). Unlike the frameshift mutants, the R323C mutant is still able to efficiently interact with tRNA substrates (Figure 4). The retention of tRNA binding by the R323C mutant is consistent with the location of the R323 residue on the interior of TRMT1 within the methyltransferase motif and outside of the putative zinc finger motif that interacts with tRNA. While the R323C mutant can still bind RNA, it exhibits a severe defect in the reconstitution of $m 2,2 \mathrm{G}$ formation in TRMT1-KO human cells. Due to the location of the R323 residue in the core of the TRMT1 methyltransferase domain, the R323C mutation could severely distort the tertiary structure of TRMT1 leading to defects in substrate orientation, SAM binding and/or catalysis.

The $\mathrm{m} 2,2 \mathrm{G}$ modification has been predicted to prevent the folding of certain nuclearencoded tRNAs into alternative conformers found in mitochondrial tRNAs (Steinberg and Cedergren 1995). Moreover, previous studies in S. cerevisiae have found that certain tRNA isoacceptors exhibit decreased accumulation in the absence of Trm1, suggesting that the $\mathrm{m} 2,2 \mathrm{G}$ modification plays a role in the proper folding of certain tRNAs (Dewe et al. 2012; Vakiloroayaei et al. 2017). Interestingly, we have found that TRMT1-deficient human cells exhibit similar steadystate levels of all tested nuclear- and mitochondrial-encoded tRNAs (Dewe et al. 2017). However, it could be possible that the $\mathrm{m} 2,2 \mathrm{G}$ modification is more important for tRNA stability and accumulation for certain human tRNA isodecoders, akin to the situation in S. cerevisiae. Thus, it will be interesting to use global profiling approaches to measure tRNA levels to determine if loss of $\mathrm{m} 2,2 \mathrm{G}$ impacts some tRNAs more than others. This will allow us to hone in on certain tRNAs that might be more dependent upon the $\mathrm{m} 2,2 \mathrm{G}$ modification for processing and stability.

The $\mathrm{m} 2,2 \mathrm{G}$ modification could also play a role in proper tRNA interaction with the ribosome to ensure efficient translation. Indeed, studies in yeast S. cerevisiae have found that Trm1 deletion mutants display alterations in ribosome profiles indicative of translation aberrations (Chou et al. 
2017). Moreover, studies in human cells have found that global translation is reduced upon ablation of TRMT1 (Dewe et al. 2017). The alterations in translation are correlated with perturbations in ROS homeostasis and heightened sensitivity to oxidative stress. Future studies using ribosome profiling in patient cells could provide insight into the biological pathways that are perturbed upon loss of the $\mathrm{m} 2,2 \mathrm{G}$ modification that contribute to the spectrum of neurodevelopmental phenotypes exhibited by individuals with TRMT1 mutations.

\section{Materials and Methods}

\section{Human subjects}

Evaluation of the affected individual and family members by a board-certified clinical geneticist included obtaining medical and family histories, clinical examination, neuroimaging and clinical laboratory investigations. After obtaining a written informed consent for enrollment in an IRB-approved project (KFSHRC RAC\#2070023), venous blood was collected in EDTA and sodium heparin tubes for DNA extraction and establishment of lymphoblastoid cell lines. All studies abide by the Declaration of Helsinki principles.

\section{In silico analysis of TRMT1 structure}

The predicted tertiary structure of human TRMT1 (NP_001129507.1) was determined using RaptorX software (http://raptorx.uchicago.edu). Structural visualization, editing, and comparison was performed using UCSF Chimera software (Pettersen et al. 2004). The arginine323 to methionine mutation was modeled by selecting the most probable rotamer conformation according to the Richardson backbone-independent rotamer library (Lovell et al. 2000). The predicted effects of the R323C mutation on TRMT1 structure was performed using DynaMut (Rodrigues et al. 2018).

\section{Plasmid constructs}

The pcDNA3.1-FLAG-TRMT1-WT and TRMT1-Q415fs expression plasmids have been described previously (Dewe et al. 2017). The pcDNA3.1-FLAG-TRMT1-R323C expression 
construct was generated by Dpnl site-directed mutagenesis. All plasmid constructs have been verified by Sanger sequencing.

\section{Tissue cell culture}

LCLs were grown to confluency and passaged in RPMI 1640 media (ThermoFisher) containing 15\% Fetal Bovine Serum, 2 mM L-alanyl-L-glutamine (GlutaMax; Gibco), and 1x penicillin/streptomycin at $37^{\circ} \mathrm{C}$ with $5 \% \mathrm{CO}_{2}$. The 293T control-WT and TRMT1-KO cells lines were previously described (Dewe et al. 2017). 293T cells lines were cultured in Dulbecco's Minimal Essential Medium (DMEM) supplemented with $10 \%$ fetal bovine serum (FBS), 1x Glutamax (Gibco), 1x penicillin and streptomycin (ThermoFisher) at $37^{\circ} \mathrm{C}$ with $5 \% \mathrm{CO}_{2}$. Cells were passaged every three days with $0.25 \%$ Trypsin. For TRMT1 re-expression experiments, pcDNA3.1 vector or pcDNA3.1-TRMT1-expression constructs were introduced into the controlWT or TRMT1-KO1 cell lines using calcium phosphate transient transfection as previously described (Dewe et al. 2017; Kingston et al. 2003). Cells were harvested 48 hours posttransfection for subsequent analysis.

\section{RNA analysis}

RNA was extracted using TRIzol LS reagent (Invitrogen). RNAs were diluted into formamide load buffer, heated to $95^{\circ} \mathrm{C}$ for 3 minutes, and fractionated on a $10 \%$ polyacrylamide, Tris-Borate-EDTA (TBE) gel containing 7M urea. Sybr Gold nucleic acid staining (Invitrogen) was conducted to identify the RNA pattern. For primer extension analysis, $1.5 \mu \mathrm{g}$ of total RNA was pre-annealed with 5'-32 $\mathrm{P}$-labeled oligonucleotide and $5 x$ hybridization buffer $(250 \mathrm{mM}$ Tris, $\mathrm{pH} 8.5$, and $300 \mathrm{mM} \mathrm{NaCl}$ ) in a total volume of $7 \mu \mathrm{l}$. The mixture was heated at $95^{\circ} \mathrm{C}$ for 3 min followed by slow cooling to $42^{\circ} \mathrm{C}$. An equal amount of extension mix consisting of avian myeloblastosis virus reverse transcriptase (Promega), 5x AMV buffer and $40 \mu \mathrm{M}$ dNTPs was added. The mixture was then incubated at $42^{\circ} \mathrm{C}$ for 1 hour and loaded on $15 \% 7 \mathrm{M}$ urea denaturing polyacrylamide gel. Gels were exposed on a phosphor screen (GE Healthcare) and scanned on a Bio-Rad 
personal molecular followed by analysis using $\mathrm{NIH}$ ImageJ software. Primer extension oligonucleotide sequences were previously described (Dewe et al. 2017).

\section{Liquid chromatography-mass spectrometry}

Total RNA from three independent flasks of wildtype and TRMT1-R323C LCLs was isolated using Trizol RNA extraction. Total RNA $(20 \mu \mathrm{g})$ was digested with nucleoside digestion mix (NEB \#M0649) at $37^{\circ} \mathrm{C}$ overnight. LC-MS analysis of digested nucleosides was analyzed as described previously (Cai et al. 2015; Dewe et al. 2017). Briefly, ribonucleosides were separated using a Hypersil GOLD ${ }^{\mathrm{TM}}$ C18 Selectivity Column (Thermo Scientific) followed by nucleoside analysis using a $Q$ Exactive Plus Hybrid Quadrupole-Orbitrap. The modification difference ratio was calculated using the $\mathrm{m} / \mathrm{z}$ intensity values of each modified nucleoside between WT and R323C-LCL mutants following normalization to the sum of intensity values for the canonical nucleosides; A, U, G and C.

\section{Protein purification and analysis.}

Cellular extract was prepared as previously described (Dewe et al. 2017). Briefly, human 293T cells were harvested by trypsinization and washed once with PBS. The cell pellet was

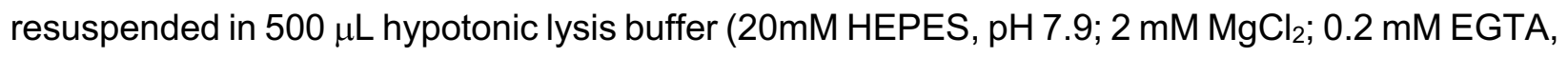
10\% glycerol, $0.1 \mathrm{mM}$ PMSF, $1 \mathrm{mM}$ DTT), incubated on ice for 5 minutes and subjected to three freeze-thaw cycles in liquid nitrogen and $37^{\circ} \mathrm{C} . \mathrm{NaCl}$ was added to extracts till the final concentration reached $400 \mathrm{mM}$. After centrifugation at $14,000 \mathrm{xg}$ for 15 minutes at $4^{\circ} \mathrm{C}$, an equal amount of hypotonic lysis buffer with $0.2 \%$ NP-40 was added to $500 \mu \mathrm{L}$ of soluble cellular extract. FLAG-tagged proteins were purified by incubating whole cell lysates from the transfected cell lines with $20 \mu \mathrm{L}$ of DYKDDDDK-Tag Monoclonal Antibody Magnetic Microbead (Syd Labs) for three hours at $4{ }^{\circ} \mathrm{C}$. Magnetic resin was washed three times in hypotonic lysis buffer with $200 \mathrm{mM} \mathrm{NaCl}$.

For protein immunoblotting, cell extracts and purified protein samples were boiled at $95^{\circ} \mathrm{C}$ for 5 minutes followed by fractionation on NuPAGE Bis-Tris polyacrylamide gels (Thermo 
Scientific). Separated proteins were transferred to Immobilon FL polyvinylidene difluoride (PVDF) membrane (Millipore) for immunoblotting. Membrane was blocked by Odyssey blocking buffer for 1 hour at room temperature followed by immunoblotting with the following antibodies: anti-FLAG epitope tag (L00018; Sigma) and actin (L00003; EMD Millipore). Proteins were detected using a 1:10000 dilution of fluorescent IRDye $800 \mathrm{CW}$ goat anti-mouse IgG (925-32210; Thermofisher) . Immunoblots were scanned using direct infrared fluorescence via the Odyssey system (LI-COR Biosciences).

\section{Acknowledgements}

We thank the study family for agreeing to participate. We thank members of the Fu Lab for helpful discussion on the manuscript. This work was supported by the Saudi Human Genome Program, and King Salman Center for Disability Research (F.S.A.), and a University of Rochester Furth Fund Award and National Science Foundation CAREER Award 1552126 to D.F..

On behalf of all authors, the corresponding author states that there is no conflict of interest.

\section{Figure Legends}

Figure 1. Characterization of a missense mutation in TRMT1 linked to ID. (A) Exon organization of the TRMT1 locus with the location of the single $\mathrm{C}>\mathrm{T}$ point mutation highlighted in red. (B) Pedigree of the family harboring the missense mutation in the TRMT1 gene and the patient that is homozygous for the mutation. (C) Patient 13DG1615 who is homozygous for the TRMT1 missense mutation who exhibits ID, epilepsy and subtle facial dysmorphism.

Figure 2. The ID-associated R323C mutation is located at a conserved position in TRMT1 and predicted to perturb protein structure. (A) Schematic of human TRMT1 with protein domains denoted; MTS (mitochondrial targeting signal), SAM-methyltransferase, and zing-finger motif. The 
location of the R323C mutation is denoted in red. (B) Protein sequence alignments of TRMT1 from human to Archaea. The R323 residue is boxed in red. (C) The structure of Pyrococcus horikoshi Trm1 (PDB: 2EJT, (Ihsanawati et al. 2008)). The location of the homologous R323 residue is highlighted in cyan. (D) Predicted structure of wildtype TRMT1 based upon in silico template-based tertiary structure prediction (Kallberg et al. 2012). The side chains of the mutated R323 and neighboring tyrosine 321 (Y321) residues are denoted in space-filling form colored cyan and beige, respectively. (E) Predicted structure of TRMT1-R323C with the R323C mutation denoted. Atoms in the side chain of R323C and Y321 that exhibit steric overlap and clash are denoted in yellow and red, respectively.

Figure 3. The ID-affected individual expressing only TRMT1-R323C exhibits a severe reduction in $\mathrm{m} 2,2, \mathrm{G}$ modification in tRNA. (A) Comparison of tRNA modification levels between R323C versus WT-LCLs. Nucleosides from digested tRNA samples were analyzed by LC-MS. Y-axis represents the log2-fold change in the levels of the indicated tRNA modification between the R323C patient and WT individual. Samples were measured in triplicate. ${ }^{* * *}, P<0.0001$ (B) Schematic of primer extension assay to monitor m2,2G in tRNAs. RT, reverse transcriptase. (C) Representative gels of primer extension assays to monitor the presence of $m 2,2 G$ in tRNA from the indicated LCLs. >, labeled oligonucleotide used for primer extension; D, dihydrouridine; m3C, 3-methylcytosine; m1G, 1-methylguanosine; *, background signal.

Figure 4. The TRMT1-R323C mutant retains RNA binding but is impaired in reconstitution of tRNA modification activity. (A) Schematic of TRMT1 domains and variants. WT, wildtype; R323C, ID-associated point mutant; Y445fs, TRMT1 variant encoded by an ID-causing frameshift mutation. (B) Immunoblot of whole cell extracts prepared from each human cell line transfected with the indicated constructs. Molecular weight in kiloDalton is denoted on the right. (C) Nucleic 
acid stain of RNAs extracted from the indicated input or purified samples after denaturing PAGE. The migration pattern of tRNAs, $5.8 \mathrm{~S}$ and $5 \mathrm{~S}$ rRNA is denoted.

Figure 5. The TRMT1-R323C mutant is impaired in reconstitution of tRNA modification activity in human cells. (A) Immunoblot of whole cell extracts prepared from each human cell line transfected with the indicated FLAG-expression constructs. Blot was probed with anti-FLAG antibody. (B) Representative primer extension assay to monitor the presence of $m 2,2 G$ in tRNA-Ala-AGC from cell lines transfected with the indicated constructs. >, labeled oligonucleotide used for primer extension; m2,2G, dimethylguanosine; D, dihydrouridine; " background signal. (C) Quantification of $\mathrm{m} 2,2 \mathrm{G}$ modification levels in tRNA-Ala-AGC. Primer extensions were performed three times and error bars represent the standard error of the mean. Comparison were performed using oneway ANOVA. *,$P<0.05 ;{ }^{* *}, P<0.01 ;{ }^{* * *}, P<0.001 ;{ }^{* * *}, P<0.0001$. 


\section{References}

Abbasi-Moheb L, Mertel S, Gonsior M, Nouri-Vahid L, Kahrizi K, Cirak S, Wieczorek D, Motazacker M, Esmaeeli-Nieh S, Cremer K, Weißmann R, Tzschach A, Garshasbi M, Abedini S, Najmabadi H, Ropers H, Sigrist S, Kuss A (2012) Mutations in NSUN2 cause autosomal-recessive intellectual disability. American journal of human genetics 90: 847855. doi: 10.1016/j.ajhg.2012.03.021

Alazami AM, Hijazi H, Al-Dosari MS, Shaheen R, Hashem A, Aldahmesh MA, Mohamed JY, Kentab A, Salih MA, Awaji A, Masoodi TA, Alkuraya FS (2013) Mutation in ADAT3, encoding adenosine deaminase acting on transfer RNA, causes intellectual disability and strabismus. J Med Genet 50: 425-30. doi: 10.1136/jmedgenet-2012-101378

Anazi S, Maddirevula S, Faqeih E, Alsedairy H, Alzahrani F, Shamseldin HE, Patel N, Hashem M, Ibrahim N, Abdulwahab F, Ewida N, Alsaif HS, Al Sharif H, Alamoudi W, Kentab A, Bashiri FA, Alnaser M, AlWadei AH, Alfadhel M, Eyaid W, Hashem A, Al Asmari A, Saleh MM, AlSaman A, Alhasan KA, Alsughayir M, Al Shammari M, Mahmoud A, Al-Hassnan ZN, Al-Husain M, Osama Khalil R, Abd El Meguid N, Masri A, Ali R, Ben-Omran T, El Fishway P, Hashish A, Ercan Sencicek A, State M, Alazami AM, Salih MA, Altassan N, Arold ST, Abouelhoda M, Wakil SM, Monies D, Shaheen R, Alkuraya FS (2017) Clinical genomics expands the morbid genome of intellectual disability and offers a high diagnostic yield. Mol Psychiatry 22: 615-624. doi: 10.1038/mp.2016.113

Angelova MT, Dimitrova DG, Dinges N, Lence T, Worpenberg L, Carre C, Roignant JY (2018) The Emerging Field of Epitranscriptomics in Neurodevelopmental and Neuronal Disorders. Front Bioeng Biotechnol 6: 46. doi: 10.3389/fbioe.2018.00046

Ayadi L, Galvanin A, Pichot F, Marchand V, Motorin Y (2019) RNA ribose methylation (2'-Omethylation): Occurrence, biosynthesis and biological functions. Biochim Biophys Acta Gene Regul Mech 1862: 253-269. doi: 10.1016/j.bbagrm.2018.11.009

Blaesius K, Abbasi AA, Tahir TH, Tietze A, Picker-Minh S, Ali G, Farooq S, Hu H, Latif Z, Khan MN, Kaindl A (2018) Mutations in the tRNA methyltransferase 1 gene TRMT1 cause congenital microcephaly, isolated inferior vermian hypoplasia and cystic leukomalacia in addition to intellectual disability. Am J Med Genet A 176: 2517-2521. doi: 10.1002/ajmg.a.38631

Blanco S, Dietmann S, Flores JV, Hussain S, Kutter C, Humphreys P, Lukk M, Lombard P, Treps L, Popis M, Kellner S, Holter SM, Garrett L, Wurst W, Becker L, Klopstock T, Fuchs H, Gailus-Durner V, Hrabe de Angelis M, Karadottir RT, Helm M, Ule J, Gleeson JG, Odom DT, Frye M (2014) Aberrant methylation of tRNAs links cellular stress to neurodevelopmental disorders. EMBO J. doi: 10.15252/embj.201489282

Buckland RA, Maule JC, Sealey PG (1996) A cluster of transfer RNA genes (TRM1, TRR3, and TRAN) on the short arm of human chromosome 6. Genomics 35: 164-71. doi: 10.1006/geno.1996.0335

Cai WM, Chionh YH, Hia F, Gu C, Kellner S, McBee ME, Ng CS, Pang YL, Prestwich EG, Lim KS, Babu IR, Begley TJ, Dedon PC (2015) A Platform for Discovery and Quantification of 
Modified Ribonucleosides in RNA: Application to Stress-Induced Reprogramming of tRNA Modifications. Methods Enzymol 560: 29-71. doi: 10.1016/bs.mie.2015.03.004

Chou HJ, Donnard E, Gustafsson HT, Garber M, Rando OJ (2017) Transcriptome-wide Analysis of Roles for tRNA Modifications in Translational Regulation. Mol Cell 68: 978-992 e4. doi: 10.1016/j.molcel.2017.11.002

Clark WC, Evans ME, Dominissini D, Zheng G, Pan T (2016) tRNA base methylation identification and quantification via high-throughput sequencing. RNA. doi: 10.1261/rna.056531.116

Copela LA, Chakshusmathi G, Sherrer RL, Wolin SL (2006) The La protein functions redundantly with tRNA modification enzymes to ensure tRNA structural stability. RNA 12: 644-54. doi: 10.1261/rna.2307206

Davarniya B, Hu H, Kahrizi K, Musante L, Fattahi Z, Hosseini M, Maqsoud F, Farajollahi R, Wienker TF, Ropers HH, Najmabadi H (2015) The Role of a Novel TRMT1 Gene Mutation and Rare GRM1 Gene Defect in Intellectual Disability in Two Azeri Families. PLoS One 10: e0129631. doi: 10.1371/journal.pone.0129631

de Brouwer APM, Abou Jamra R, Kortel N, Soyris C, Polla DL, Safra M, Zisso A, Powell CA, Rebelo-Guiomar P, Dinges N, Morin V, Stock M, Hussain M, Shahzad M, Riazuddin S, Ahmed ZM, Pfundt R, Schwarz F, de Boer L, Reis A, Grozeva D, Raymond FL, Riazuddin S, Koolen DA, Minczuk M, Roignant JY, van Bokhoven H, Schwartz S (2018) Variants in PUS7 Cause Intellectual Disability with Speech Delay, Microcephaly, Short Stature, and Aggressive Behavior. Am J Hum Genet 103: 1045-1052. doi: 10.1016/j.ajhg.2018.10.026

Dewe JM, Fuller BL, Lentini JM, Kellner SM, Fu D (2017) TRMT1-Catalyzed tRNA Modifications Are Required for Redox Homeostasis To Ensure Proper Cellular Proliferation and Oxidative Stress Survival. Mol Cell Biol 37. doi: 10.1128/MCB.00214-17

Dewe JM, Whipple JM, Chernyakov I, Jaramillo LN, Phizicky EM (2012) The yeast rapid tRNA decay pathway competes with elongation factor $1 \mathrm{~A}$ for substrate tRNAs and acts on tRNAs lacking one or more of several modifications. RNA 18: 1886-96. doi: 10.1261/rna.033654.112

El Yacoubi B, Bailly M, de Crecy-Lagard V (2012) Biosynthesis and function of posttranscriptional modifications of transfer RNAs. Annu Rev Genet 46: 69-95. doi: 10.1146/annurev-genet110711-155641

El-Hattab AW, Saleh MA, Hashem A, Al-Owain M, Asmari AA, Rabei H, Abdelraouf H, Hashem M, Alazami AM, Patel N, Shaheen R, Faqeih EA, Alkuraya FS (2016) ADAT3-related intellectual disability: Further delineation of the phenotype. Am J Med Genet A 170A: 1142-7. doi: 10.1002/ajmg.a.37578

Ellis SR, Hopper AK, Martin NC (1987) Amino-terminal extension generated from an upstream AUG codon is not required for mitochondrial import of yeast N2,N2-dimethylguanosinespecific tRNA methyltransferase. Proc Natl Acad Sci U S A 84: 5172-6.

Ellis SR, Hopper AK, Martin NC (1989) Amino-terminal extension generated from an upstream AUG codon increases the efficiency of mitochondrial import of yeast N2,N2dimethylguanosine-specific tRNA methyltransferases. Mol Cell Biol 9: 1611-20. 
Ellis SR, Morales MJ, Li JM, Hopper AK, Martin NC (1986) Isolation and characterization of the TRM1 locus, a gene essential for the N2,N2-dimethylguanosine modification of both mitochondrial and cytoplasmic tRNA in Saccharomyces cerevisiae. J Biol Chem 261: 9703-9.

Frye M, Harada BT, Behm M, He C (2018) RNA modifications modulate gene expression during development. Science 361: 1346-1349. doi: 10.1126/science.aau1646

Hopper AK, Furukawa AH, Pham HD, Martin NC (1982) Defects in modification of cytoplasmic and mitochondrial transfer RNAs are caused by single nuclear mutations. Cell 28: 543-50.

Hori H (2014) Methylated nucleosides in tRNA and tRNA methyltransferases. Front Genet 5: 144. doi: 10.3389/fgene.2014.00144

Ihsanawati, Nishimoto M, Higashijima K, Shirouzu M, Grosjean H, Bessho Y, Yokoyama S (2008) Crystal structure of tRNA N2,N2-guanosine dimethyltransferase Trm1 from Pyrococcus horikoshii. J Mol Biol 383: 871-84. doi: 10.1016/j.jmb.2008.08.068

Kallberg M, Wang H, Wang S, Peng J, Wang Z, Lu H, Xu J (2012) Template-based protein structure modeling using the RaptorX web server. Nat Protoc 7: 1511-22. doi: 10.1038/nprot.2012.085

Khan M, Rafiq M, Noor A, Hussain S, Flores J, Rupp V, Vincent A, Malli R, Ali G, Khan F, Ishak G, Doherty D, Weksberg R, Ayub M, Windpassinger C, Ibrahim S, Frye M, Ansar M, Vincent J (2012) Mutation in NSUN2, which encodes an RNA methyltransferase, causes autosomal-recessive intellectual disability. American journal of human genetics 90: 856863. doi: 10.1016/j.ajhg.2012.03.023

Kingston RE, Chen CA, Rose JK (2003) Calcium phosphate transfection. Curr Protoc Mol Biol Chapter 9: Unit 9 1. doi: 10.1002/0471142727.mb0901s63

Komara M, Al-Shamsi AM, Ben-Salem S, Ali BR, Al-Gazali L (2015) A Novel Single-Nucleotide Deletion (c.1020delA) in NSUN2 Causes Intellectual Disability in an Emirati Child. J Mol Neurosci 57: 393-9. doi: 10.1007/s12031-015-0592-8

Liu J, Straby KB (2000) The human tRNA(m(2)(2)G(26))dimethyltransferase: functional expression and characterization of a cloned hTRM1 gene. Nucleic Acids Res 28: 344551.

Lovell SC, Word JM, Richardson JS, Richardson DC (2000) The penultimate rotamer library. Proteins 40: 389-408.

Martinez FJ, Lee JH, Lee JE, Blanco S, Nickerson E, Gabriel S, Frye M, AI-Gazali L, Gleeson JG (2012) Whole exome sequencing identifies a splicing mutation in NSUN2 as a cause of a Dubowitz-like syndrome. J Med Genet 49: 380-5. doi: 10.1136/jmedgenet-2011-100686

Monies D, Abouelhoda M, AlSayed M, Alhassnan Z, Alotaibi M, Kayyali H, Al-Owain M, Shah A, Rahbeeni Z, Al-Muhaizea MA, Alzaidan HI, Cupler E, Bohlega S, Faqeih E, Faden M, Alyounes B, Jaroudi D, Goljan E, Elbardisy H, Akilan A, Albar R, Aldhalaan H, Gulab S, Chedrawi A, Al Saud BK, Kurdi W, Makhseed N, Alqasim T, El Khashab HY, Al-Mousa H, Alhashem A, Kanaan I, Algoufi T, Alsaleem K, Basha TA, Al-Murshedi F, Khan S, Al-Kindy 
A, Alnemer M, Al-Hajjar S, Alyamani S, Aldhekri H, Al-Mehaidib A, Arnaout R, Dabbagh O, Shagrani M, Broering D, Tulbah M, Alqassmi A, Almugbel M, AIQuaiz M, Alsaman A, Al-Thihli K, Sulaiman RA, Al-Dekhail W, Alsaegh A, Bashiri FA, Qari A, Alhomadi S, Alkuraya $\mathrm{H}$, Alsebayel M, Hamad MH, Szonyi L, Abaalkhail F, Al-Mayouf SM, Almojalli $\mathrm{H}$, Alqadi KS, Elsiesy H, Shuaib TM, Seidahmed MZ, Abosoudah I, Akleh H, AIGhonaium A, Alkharfy TM, Al Mutairi F, Eyaid W, Alshanbary A, Sheikh FR, Alsohaibani FI, Alsonbul A, Al Tala S, Balkhy S, Bassiouni R, Alenizi AS, Hussein MH, Hassan S, Khalil M, Tabarki B, Alshahwan S, Oshi A, Sabr Y, Alsaadoun S, Salih MA, Mohamed S, Sultana H, Tamim A, El-Haj M, Alshahrani S, Bubshait DK, Alfadhel M, et al. (2017) The landscape of genetic diseases in Saudi Arabia based on the first 1000 diagnostic panels and exomes. Hum Genet 136: 921-939. doi: 10.1007/s00439-017-1821-8

Monies D, Vagbo CB, Al-Owain M, Alhomaidi S, Alkuraya FS (2019) Recessive Truncating Mutations in ALKBH8 Cause Intellectual Disability and Severe Impairment of Wobble Uridine Modification. Am J Hum Genet 104: 1202-1209. doi: 10.1016/j.ajhg.2019.03.026

Najmabadi H, Hu H, Garshasbi M, Zemojtel T, Abedini S, Chen W, Hosseini M, Behjati F, Haas S, Jamali P, Zecha A, Mohseni M, Püttmann L, Vahid L, Jensen C, Moheb L, Bienek M, Larti F, Mueller I, Weissmann R, Darvish H, Wrogemann K, Hadavi V, Lipkowitz B, Esmaeeli-Nieh S, Wieczorek D, Kariminejad R, Firouzabadi S, Cohen M, Fattahi Z, Rost I, Mojahedi F, Hertzberg C, Dehghan A, Rajab A, Banavandi M, Hoffer J, Falah M, Musante L, Kalscheuer V, Ullmann R, Kuss A, Tzschach A, Kahrizi K, Ropers H (2011) Deep sequencing reveals 50 novel genes for recessive cognitive disorders. Nature 478: 57-63. doi: 10.1038/nature10423

Ontiveros RJ, Stoute J, Liu KF (2019) The chemical diversity of RNA modifications. Biochem J 476: 1227-1245. doi: 10.1042/BCJ20180445

Pettersen EF, Goddard TD, Huang CC, Couch GS, Greenblatt DM, Meng EC, Ferrin TE (2004) UCSF Chimera--a visualization system for exploratory research and analysis. J Comput Chem 25: 1605-12. doi: 10.1002/jcc.20084

Ramos J, Fu D (2018) The emerging impact of tRNA modifications in the brain and nervous system. Biochim Biophys Acta Gene Regul Mech. doi: 10.1016/j.bbagrm.2018.11.007

Ramos J, Han L, Li Y, Hagelskamp F, Kellner SM, Alkuraya FS, Phizicky EM, Fu D (2019) Formation of tRNA wobble inosine in humans is disrupted by a millennia-old mutation causing intellectual disability. Mol Cell Biol. doi: 10.1128/MCB.00203-19

Ranjan N, Leidel SA (2019) The epitranscriptome in translation regulation: mRNA and tRNA modifications as the two sides of the same coin? FEBS Lett 593: 1483-1493. doi: 10.1002/1873-3468.13491

Rodrigues CH, Pires DE, Ascher DB (2018) DynaMut: predicting the impact of mutations on protein conformation, flexibility and stability. Nucleic Acids Res 46: W350-W355. doi: 10.1093/nar/gky300

Shaheen R, Abdel-Salam GM, Guy MP, Alomar R, Abdel-Hamid MS, Afifi HH, Ismail SI, Emam BA, Phizicky EM, Alkuraya FS (2015) Mutation in WDR4 impairs tRNA m(7)G46 methylation and causes a distinct form of microcephalic primordial dwarfism. Genome Biol 16: 210. doi: 10.1186/s13059-015-0779-x 
Shaheen R, Al-Salam Z, El-Hattab AW, Alkuraya FS (2016a) The syndrome dysmorphic facies, renal agenesis, ambiguous genitalia, microcephaly, polydactyly and lissencephaly (DREAM-PL): Report of two additional patients. Am J Med Genet A 170: 3222-3226. doi: 10.1002/ajmg.a.37877

Shaheen R, Han L, Faqeih E, Ewida N, Alobeid E, Phizicky EM, Alkuraya FS (2016b) A homozygous truncating mutation in PUS3 expands the role of tRNA modification in normal cognition. Hum Genet 135: 707-13. doi: 10.1007/s00439-016-1665-7

Shaheen R, Mark P, Prevost CT, AlKindi A, Alhag A, Estwani F, Al-Sheddi T, Alobeid E, Alenazi MM, Ewida N, Ibrahim N, Hashem M, Abdulwahab F, Bryant EM, Spinelli E, Millichap J, Barnett SS, Kearney HM, Accogli A, Scala M, Capra V, Nigro V, Fu D, Alkuraya FS (2019a) Biallelic variants in CTU2 cause DREAM-PL syndrome and impair thiolation of tRNA wobble U34. Hum Mutat. doi: 10.1002/humu.23870

Shaheen R, Tasak M, Maddirevula S, Abdel-Salam GMH, Sayed ISM, Alazami AM, Al-Sheddi T, Alobeid E, Phizicky EM, Alkuraya FS (2019b) PUS7 mutations impair pseudouridylation in humans and cause intellectual disability and microcephaly. Hum Genet 138: 231-239. doi: 10.1007/s00439-019-01980-3

Steinberg S, Cedergren R (1995) A correlation between N2-dimethylguanosine presence and alternate tRNA conformers. RNA 1: 886-91.

Suzuki T, Suzuki T (2014) A complete landscape of post-transcriptional modifications in mammalian mitochondrial tRNAs. Nucleic Acids Res 42: 7346-57. doi: 10.1093/nar/gku390

Vakiloroayaei A, Shah NS, Oeffinger M, Bayfield MA (2017) The RNA chaperone La promotes pre-tRNA maturation via indiscriminate binding of both native and misfolded targets. Nucleic Acids Res 45: 11341-11355. doi: 10.1093/nar/gkx764

Vauti F, Goller T, Beine R, Becker L, Klopstock T, Hölter S, Wurst W, Fuchs H, Gailus-Durner V, de Angelis M, Arnold $\mathrm{H}-\mathrm{H}$ (2007) The mouse Trm1-like gene is expressed in neural tissues and plays a role in motor coordination and exploratory behaviour. Gene 389: 174-185. doi: 10.1016/j.gene.2006.11.004 


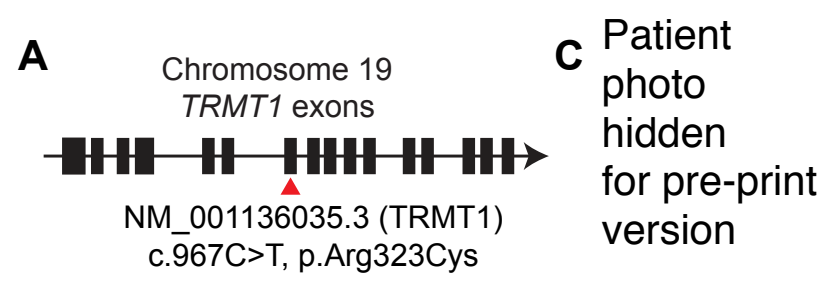

B

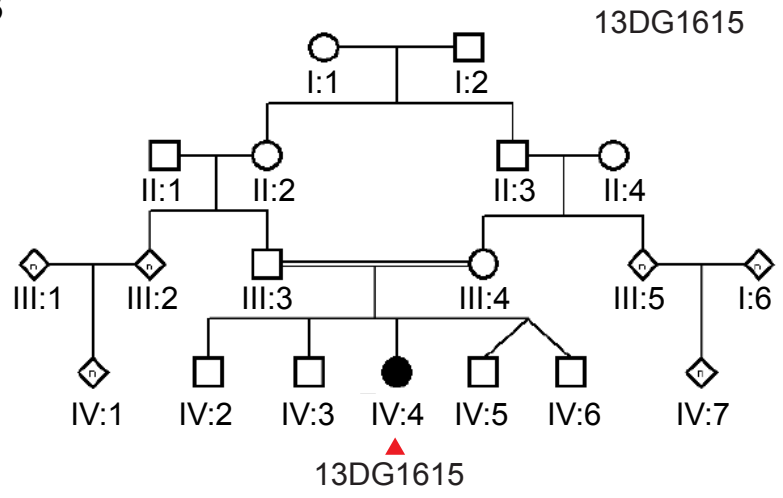

Figure 1. Characterization of a missense mutation in TRMT1 linked to ID. (A) Exon organization of the TRMT1 locus with the location of the single C>T point mutation highlighted in red. (B) Pedigree of the family harboring the missense mutation in the TRMT1 gene and the patient that is homozygous for the mutation. (C) Patient 13DG1615 who is homozygous for the TRMT1 missense mutation who exhibits ID, epilepsy and subtle facial dysmorphism. 
A

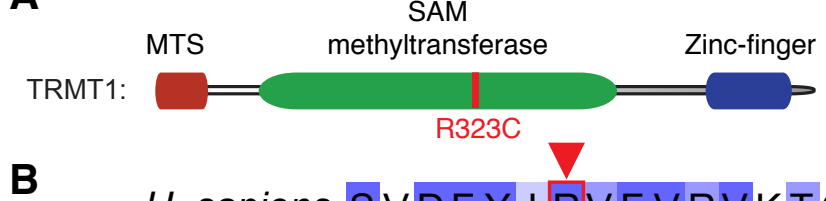

H. sapiens SVDFY I RVFVRVKTG

M. musculus SADF YVRVFVRVFTG

$X$. laevis SADFY I RVF VRVFTG

D. rerio SADFY I RVFVRVFTG

D. melanogaster SADF Y I R I FVRVY VG

C. elegans SVDF Y VRVFVRVHTG

S. cerevisiae S I DFYVRVFVKVKTS

$P$. furiosus $\mathrm{YKDHYFRAFVKLKDG}$
C

P. horikoshii Trm1

PDB: 2EJT

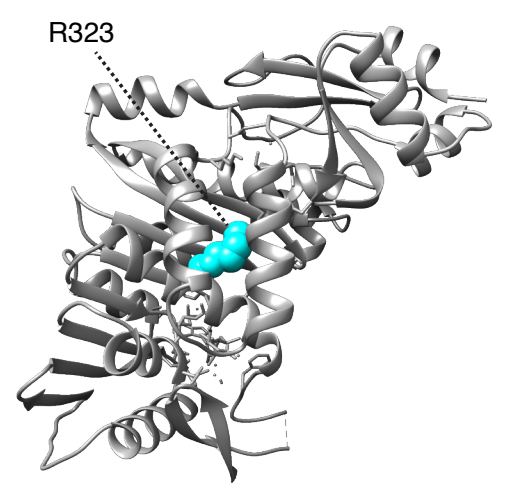

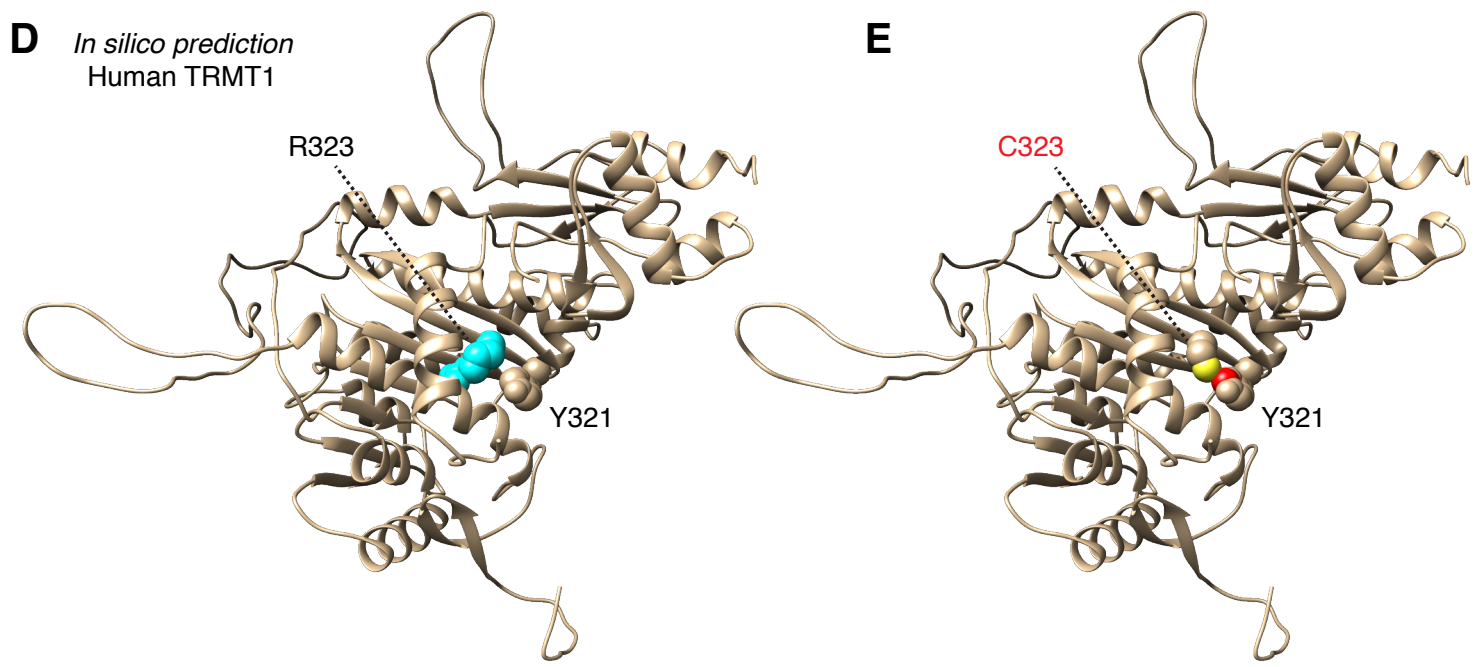

Figure 2. The ID-associated R323C mutation is located at a conserved position in TRMT1 and predicted to perturb protein structure. (A) Schematic of human TRMT1 with protein domains denoted; MTS (mitochondrial targeting signal), SAM-methyltransferase, and zing-finger motif. The location of the R323C mutation is denoted in red. (B) Protein sequence alignments of TRMT1 from human to Archaea. The R323 residue is boxed in red. (C) The structure of Pyrococcus horikoshi Trm1 (PDB: 2EJT, (Ihsanawati et al. 2008)). The location of the homologous $\mathrm{R} 323$ residue is highlighted in cyan. (D) Predicted structure of wildtype TRMT1 based upon in silico template-based tertiary structure prediction (Kallberg et al. 2012). The side chains of the mutated R323 and neighboring tyrosine 321 (Y321) residues are denoted in space-filling form colored cyan and beige, respectively. (E) Predicted structure of TRMT1-R323C with the R323C mutation denoted. Atoms in the side chain of R323C and Y321 that exhibit steric overlap and clash are denoted in yellow and red, respectively. 


\section{A}

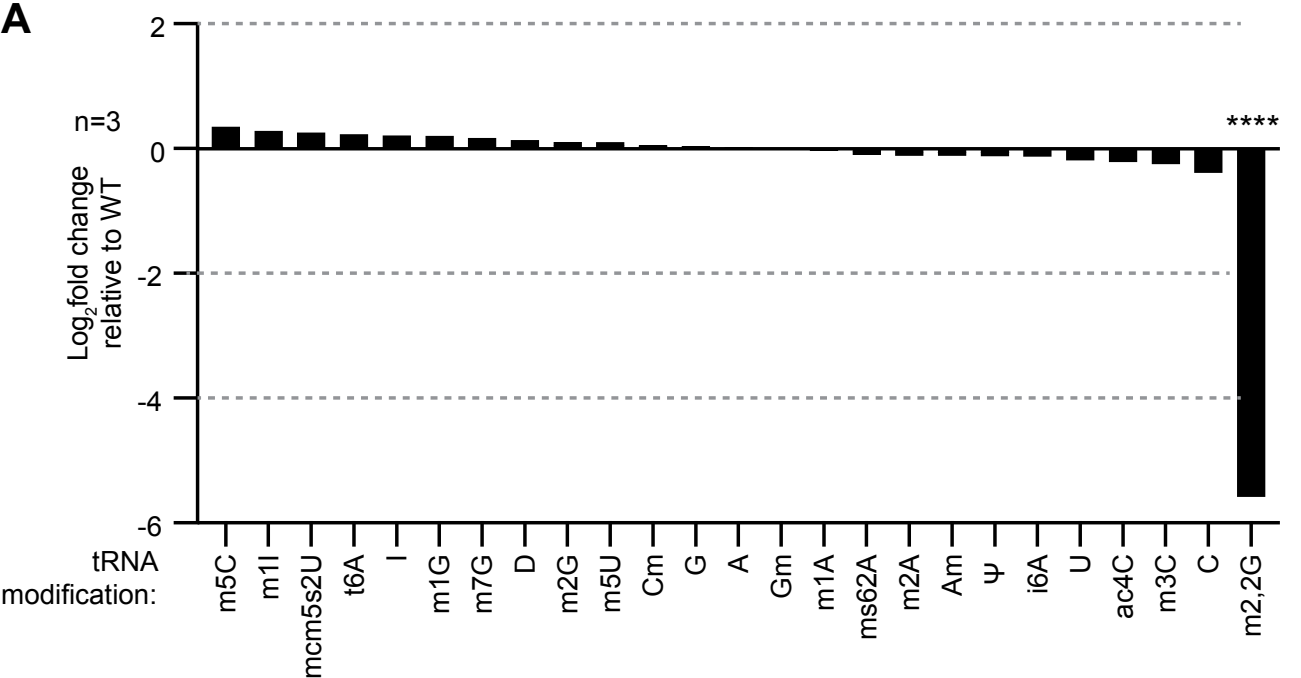

B

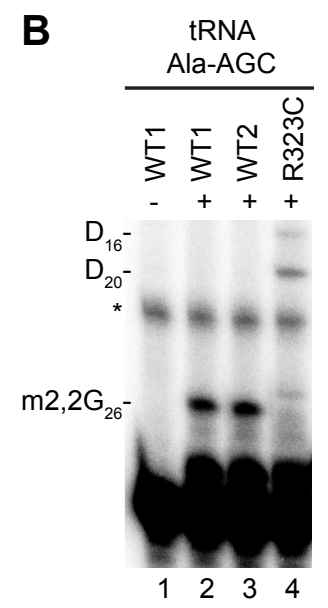

tRNA
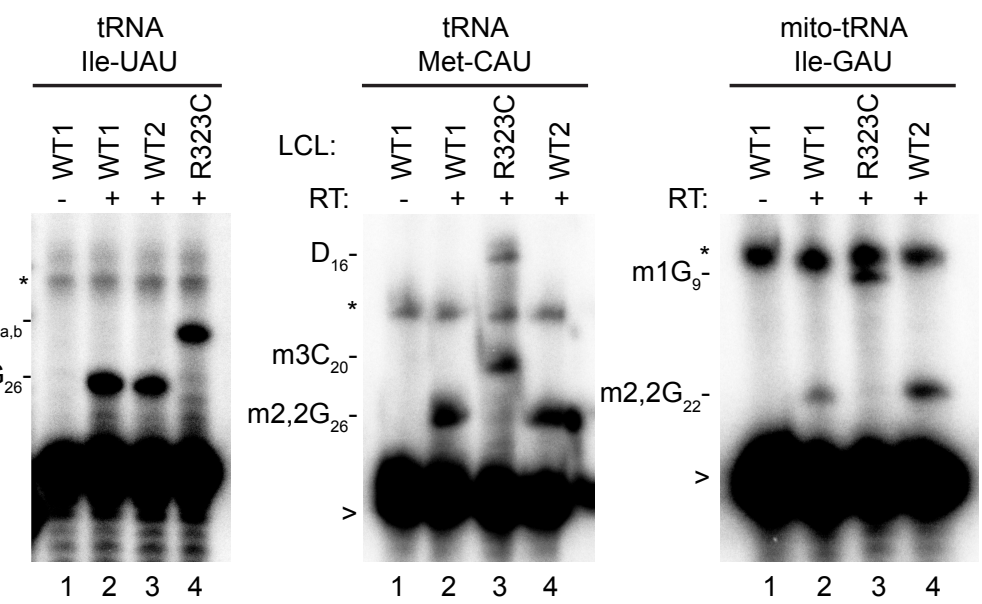

Figure 3. The ID-affected individual expressing only TRMT1-R323C exhibits a severe reduction in $\mathrm{m} 2,2, \mathrm{G}$ modification in tRNA. (A) Comparison of tRNA modification levels between R323C versus WT-LCLs. Nucleosides from digested tRNA samples were analyzed by LC-MS. Y-axis represents the log2-fold change in the levels of the indicated tRNA modification between the R323C patient and WT individual. Samples were measured in triplicate. ${ }^{* * * *}, P<0.0001$ (B) Schematic of primer extension assay to monitor m2,2G in tRNAs. RT, reverse transcriptase. (C) Representative gels of primer extension assays to monitor the presence of $m 2,2 \mathrm{G}$ in tRNA from the indicated LCLs. >, labeled oligonucleotide used for primer extension; D, dihydrouridine; m3C, 3-methylcytosine; m1G, 1-methylguanosine; *, background signal. 


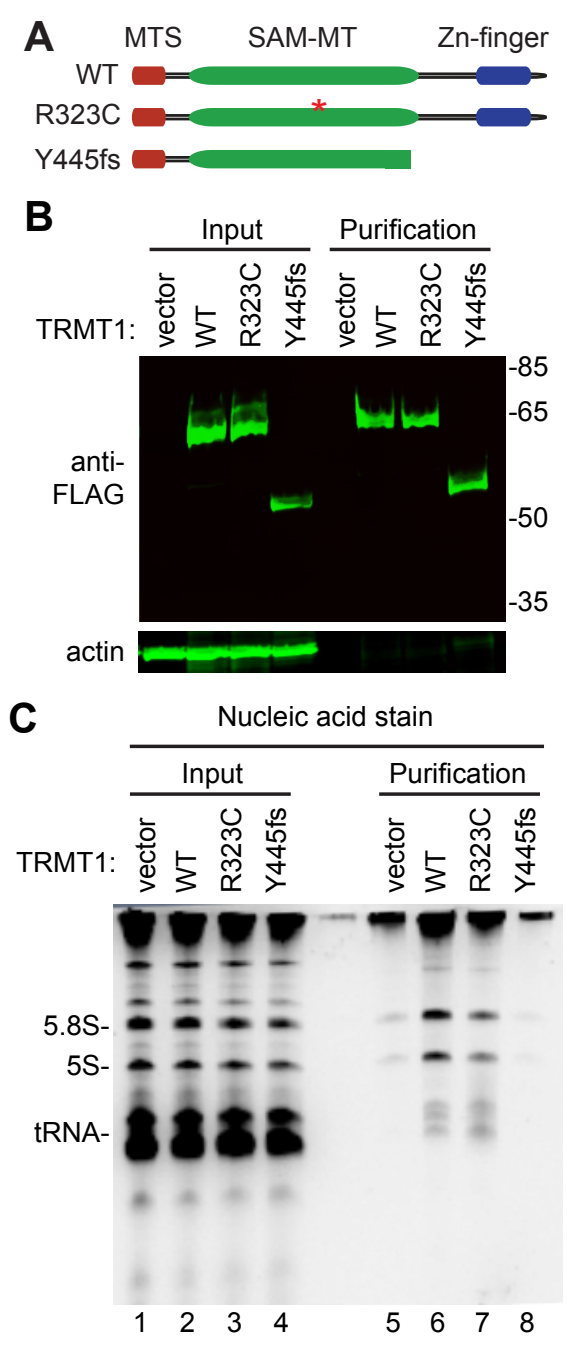

Figure 4. The TRMT1-R323C mutant retains RNA binding but is impaired in reconstitution of tRNA modification activity. (A) Schematic of TRMT1 domains and variants. WT, wildtype; R323C, ID-associated point mutant; Y445fs, TRMT1 variant encoded by an ID-causing frameshift mutation. (B) Immunoblot of whole cell extracts prepared from each human cell line transfected with the indicated constructs. Molecular weight in kiloDalton is denoted on the right. (C) Nucleic acid stain of RNAs extracted from the indicated input or purified samples after denaturing PAGE. The migration pattern of tRNAs, 5.8S and 5S rRNA is denoted. 

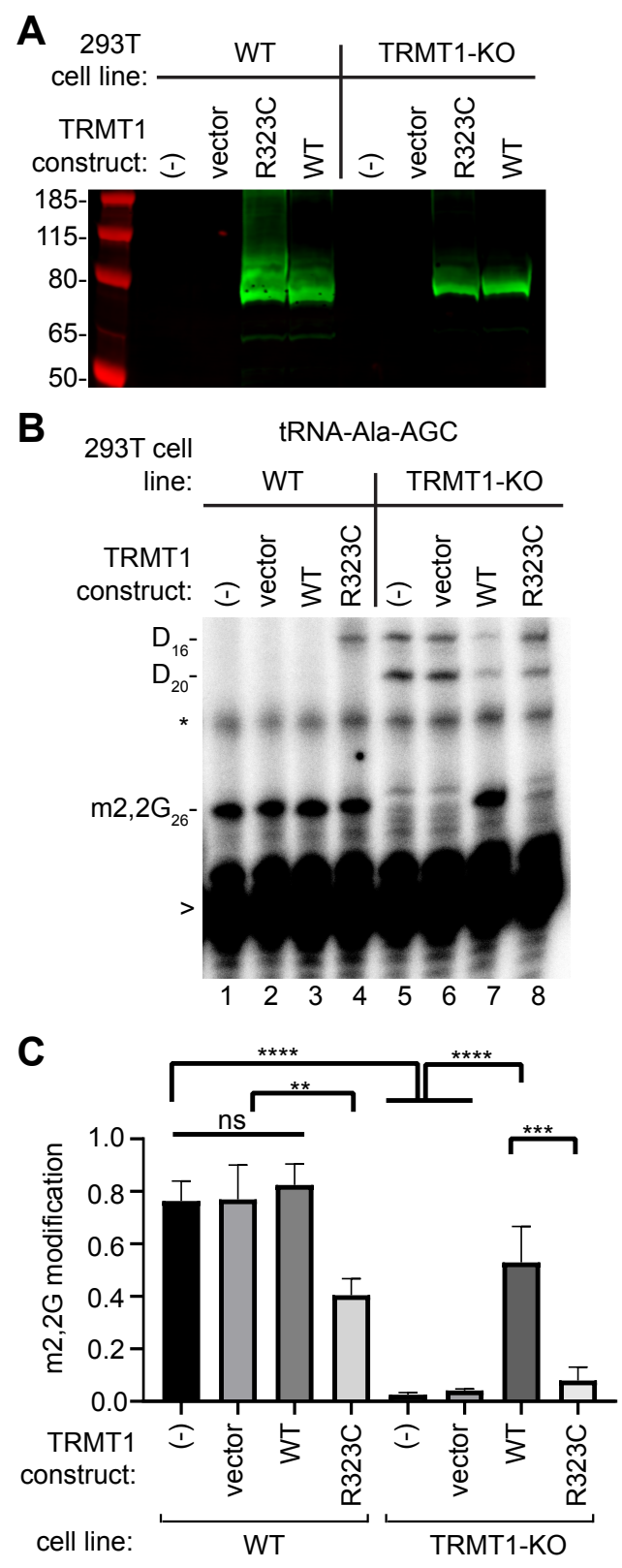

Figure 5. The TRMT1-R323C mutant is impaired in reconstitution of tRNA modification activity in human cells. (A) Immunoblot of whole cell extracts prepared from each human cell line transfected with the indicated FLAG-expression constructs. Blot was probed with anti-FLAG antibody. (B) Representative primer extension assay to monitor the presence of $m 2,2 G$ in tRNA-Ala-AGC from cell lines transfected with the indicated constructs. >, labeled oligonucleotide used for primer extension; $\mathrm{m} 2,2 \mathrm{G}$, dimethylguanosine; $\mathrm{D}$, dihydrouridine; * background signal. (C) Quantification of $\mathrm{m} 2,2 \mathrm{G}$ modification levels in tRNA-Ala-AGC. Primer extensions were performed three times and error bars represent the standard error of the mean. Comparison were performed using one-way ANOVA. * $\mathrm{P}<0.05 ;{ }^{* *}, \mathrm{P}<$ $0.01 ;{ }^{* * *}, \mathrm{P}<0.001 ;{ }^{* * *}, \mathrm{P}<0.0001$. 